\title{
Les navires
}

\section{non conventionnels:}

réalisalions actuelles

\section{êt développements envisageables}

\author{
Jean Le Tallec \\ Ingénıeur Principal de l'Armement \\ Direction Technique des Constructions Navales
}

Il n'est pas possible de donner une définition technique satisfaisante valable pour l'ensemble des navires dits non conventionnels. L'aéroglisseur amphibie commence à être bien connu en France avec les services transmanche des "hovercrafts" britanniques et la prochaine mise en service de l'aéroglisseur $N 500$. La présente communication est relative aux autres types de navires non conventionnels et à leurs développements possibles à moyen et long terme.

L'ensemble des véhicules marins classés dans cette famille se caractérise essentiellement comme une tentative de progrès dans deux domaines où la marine classique est limitée : la vitesse et la tenue à la mer.

Le navire classique est limité en vitesse : 35 à 40 nœuds est un seuil extrême au-delà duquel les puissances à installer deviennent excessives ; ce seuil est également celui de la propulsion par hélice marine subcavitante en raison des contraintes dues à la cavitation.

Cette vitesse maximale décroît très rapidement avec l'état de la mer. Les causes en sont variées : augmentation de la résistance à la marche, conditions de fonctionnement dégradées pour l'hélice, réduction délibérée de la vitesse pour ménager la structure et diminuer les risques de "slamming". Un navire déplaçant 4000 tonnes, ayant une vitesse maximale de 30 nœuds voit sa vitesse tomber à 15 nœuds environ par mer force 5 (vagues de 3 mètres crête à creux). Les accélérations subies par le navire représentent également une gêne pour la mise en œuvre d'armes ou le confort de l'équipage et des passagers.

Le navire non conventionnel est une tentative de progrès soit sur la vitesse, soit sur la tenue à la mer, soit sur ces deux aspects à la fois.

La recherche de vitesses élevées conduit à des puissances propulsives rapportées au déplacement beaucoup plus élevées que celles auxquelles on est habitué en cons. truction navale. Ceci est illustré dans la figure 1 pour plusieurs véhicules déplaçant 2000 tonnes, par la finesse généralisée $f^{*}$ à la vitesse maximale pour un état de mer force 2. (La finesse généralisée est le rapport déplacement $x$ vitesse/puissance installée).

\begin{tabular}{|c|c|c|c|}
\hline Type & $\begin{array}{c}\text { Navire } \\
\text { classique }\end{array}$ & Hyơroptère & $\begin{array}{c}\text { Aéroglisseur à } \\
\text { grilles latérales }\end{array}$ \\
\hline $\begin{array}{c}V \text { max mer } \\
\text { force 2 (nceuds) }\end{array}$ & 25 & 55 & 75 \\
\hline$f^{*}$ & 30 & 9 & 6 \\
\hline
\end{tabular}

Figure 1

Les puissances à installer sur des navires non conventionnels de déplacement élevé (plus de 1000 tonnes) sont donc très importantes. Un hydroptère de 2000 tonnes et de vitesse maximale 55 nouds exige un peu moins de $60 \mathrm{MW}$. Un aéroglisseur à quilles latérales de 2000 tonnes et de vitesse maximale 85 ncuds demande, pour assurer la propulsion et la sustentation de 130 à $150 \mathrm{MW}$.

La recherche de vitesses entre 50 et 90 nœuds et parallèlement d'une autonomie en rapport avec la taille du véhicule conđuit à un poids "appareil propulsif + carburant" très élevé : on conçoit qu'outre les problèmes propres à la réalisation de tels appareils propulsifs, les navires non conventionnels posent des problèmes particulièrement ardus de devis de poids qui rendent inéluctable le recours àn des techniques intermédiaires entre la construction aéronautique et la construction navale pour leur réalisation.

\section{Les différents types de navires non conventionnels}

Un seul de ces véhicules est à sustentation hydrostatique, comme les navires classiques : il s'agit du bâtiment 
de surface à coques immergées (BSCI). Tous les autres font appel à des moyens de sustentation statiques ou dynamiques permettant de déjauger complètement ou presque le véhicule.

L'hydroptère est à sustentation hydrodynamique (ailes portantes immergées).

Les véhicules à coussin d'air sont à sustentation aérostatique ; deux sous-types sont à distinguer : ceux qui n'ont aucun contact avec l'eau, les aéroglisseurs à jupes souples (AJS) et ceux qui ne sont pas amphibies, les aéroglisseurs à quilles latérales (AQL).

A partir des vitesses de l'ordre de 200 ncuds, la pression dynamique devient suffisante pour envisager des appareils à sustentation aérodynamique (ASAD). On aboutit en fait à un véritable aéronef volant en effet de sol, avec ou sans contact permanent avec l'eau.

\section{Le bôtiment de suriface à coques immergées (BSCI)}

Le BSCI (fig. 2) est un catamaran dont les deux coques sont des corps fuselés profondément immergés, reliés au pont par deux plans minces.
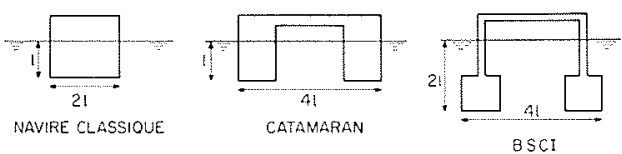

Figure 2

Initialement, l'ambition du BSCI était de réaliser un gain a la fois sur la vitesse et sur la tranquilité de plateforme. La trainée de vagues est effectivement moindre que pour un navire classique en raison de la minceur des parois verticales et de l'immersion des coques. Par contre, la trainée visqueuse est largement augmentée du fait de la surface très grande des cuvres vives. Au total, le BSCI n'est supérieur au navire classique qu'à vitesse élevée, pour des degrés de vitesse $V / 6 \sqrt{\Delta} 5$ ( $V$ étant la vitesse en $\mathrm{m} / \mathrm{s}$ et $\Delta$ le déplacement en tonnes).

Aux vitesses faibles la trainée du BSCI est supérieure à celle du navire classique. Pour dépasser 40 nœuds, les puissances deviennent très élevées : $75 \mathrm{MW}$ sont nécessaires pour un BSCI de 4000 tonnes et de 42 ncuds. Cette formule pose par ailleurs des problèmes d'aménagement : le volume disponible est réduit et rend difficile l'implantation d'une forte motorisation. En fait, les projets récents envisagent des vitesses assez modestes (30 a 35 nœuds) : le poids de structure est très élevé, $40 \%$ du déplacement contre $30 \%$ pour un navire classique : l'utilisation d'alliages légers pourrait permettre un gain sur la charge marchande.

Le BSCI pose des problèmes de stabilité dynamique qui peuvent être résolus soit par des empennages (au détriment de la trainée), soit par un pilotage par ailerons actifs.

L'avantage le plus manifeste du BSCI est sa tranquilité de plate-forme : l'impression des coques les rend moins sensibles aux sollicitations de la houle.
Le rappel statique est faible en raison de la surface de flottaison réduite, ce qui permet de déplacer les fréquences de rencontre gênantes. Le BSCI est remarquablement tranquille pour des longueurs d'onde de houle voisine de sa longueur, ce qui est en général le cas le plus sévère pour les navires classiques.

En contrepartie, sa tranquillité de plate-forme se dégrade pour les houles très longues ( 4 fois sa longueur). La recherche d'une excellente tenue à la mer pour tout cap et tout état de mer nécessite vraisemblablement un pilotage actif, ce qui fait perdre l'avantage de rusticité revendiqué initialement par cette formule.

Une réalisation à citer est un véhicule probatoire, le SSP, réalisé aux Etats-Unis (190 tonnes ; 25 nœuds ; $\mathrm{L}=30 \mathrm{~m} ; \ell=15 \mathrm{~m}$ ).

\section{L'hydroptère}

Le problème majeur rencontré par les pionniers de cette formule (fig. 3) est la stabilité. Il a été résolu sur les premiers véhicules opérationnels (hydroptère de $1^{\text {ère }}$ genération -- photo 1) par des ailes en $V$ perçant la surface libre. La surface alaire est ainsi variable en fonction de l'attitude de l'engin et permet de disposer de forces de rappel très importantes. La contrepartie de cette très grande raideur est une extrême sensibilité à la houle.
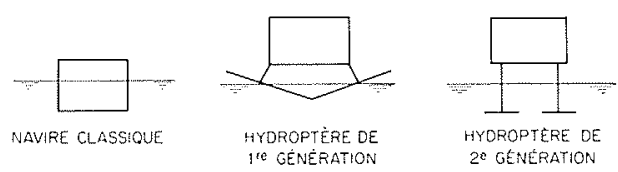

Figure 3

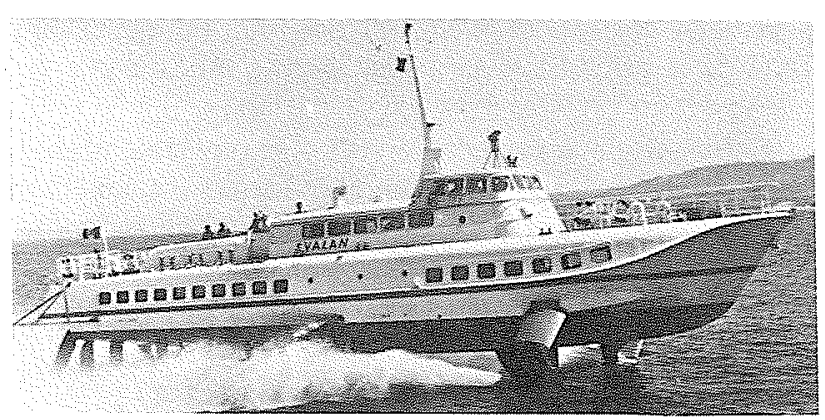

Photo 1 - Hydroptère de $1^{\text {ère }}$ génération (photo Chantiers Rodriguez).

L'hydroptère de deuxième génération a au contraire ses ailes totalement immergées. Le seul effet de rappel est une diminution de portance lorsqu'on se rapproche de la surface libre : il est toutefois insuffisant, l'hydroptère de deuxième génération est en limite de stabilité ou franchement instable naturellement. Son pilotage ne peut être qu'automatique en raison des fréquences de rencontre avec la houle : le pilotage d'un véhicule de 
200 tonnes - 50 nœuds doit tenir compte de fréquences allant jusqu'à $5 \mathrm{H}_{\mathrm{Z}}$. Vu le domaine de vol très étroit dans le plan vertical, on demande également une très grande précision a ce pilotage. Son objectif est double : limiter les accélérations verticales à des niveaux faibles $(5 / 100 \mathrm{~g})$ et garantir une attitude sûre dans tous les cas (cap et état de mer rencontré) par rapport à la surface libre.

Deux alternatives sont envisageables pour ce second objectif. On peut chercher à ne pas dégrader la tenue aux accélérations, en essayant de tenir une hauteur de vol commandée, une gite et une assiette nulle le plus strictement possible : dans ce cas l'écrêtage des vagues les plus hautes ou,phénomène plus grave, l'émergence d'une aile peuvent se produire. On peut chercher à suivre partiellement la surface de la mer pour améliorer la sûreté, en dégradant la tenue. Ceci exige une très grande confiance dans les senseurs d'attitude (détecteurs de hauteur en particulies) : des techniques inspirées du pilotage autoadaptatif, d'une prédiction à court terme du profil de houle sont à étudier dans ce sens pour améliorer encore les caractéristiques tout temps de l'hydroptère.

Deux grandes classes de pilotage sont actuellement proposées : le pilotage par braquage de toute l'aile (l'incidence hydrodynamique restant pratiquement constante dans ce cas) et le pilotage par braquage d'un volet. La première solution a l'avantage d'être moins sensible aux cavitations parasites et de permettre de maintenir la vitesse maximale pour tous les états de mer franchissables. La seconde permet de résoudre de façon plus aisée les problèmes de puissance hydraulique mises en jeu par le pilotage. Les servocommandes posent des problèmes d'encombrement, compte tenu des efforts et débits mis en jeu et des redondances nécessaires, les nacelles de raccordement entre aile et jambe devant être réduites au volume minimum.

La cavitation est le phénomène déterminant pour la vitesse maximale : les hydroptères à ailes subcavitantes, c'est-à-dire la quasi-totalité des appareils réalisés à ce jour, sont limités à 55 nœuds.

Deux réalisations américaines ont atteint le stade opérationnel :

- le PHM OTAN, construit en petite série par Boeing (photo 2) a un déplacement de 235 tonnes, une longueur de 40 mètres et une vitesse maximale de

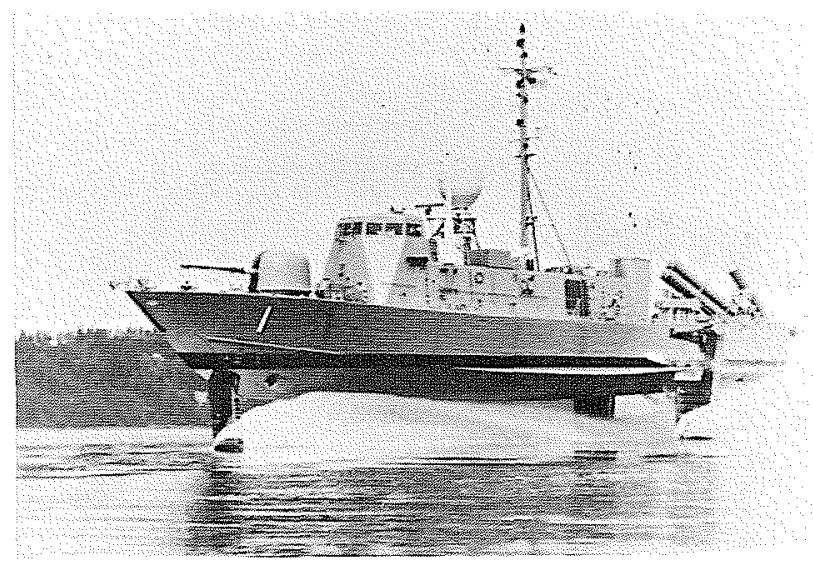

Photo 2 - Hydroptère de $2^{\mathrm{e}}$ génération, Boeing PHM "Pegasus" (photo US Navy).

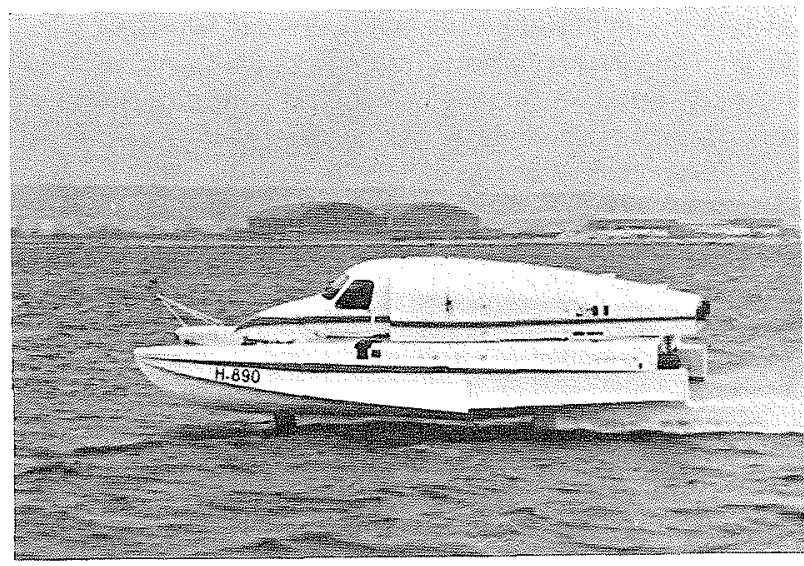

Photo 3 - Plateforme expérimentale H 890 (photo SNIAS).

50 nœuds. Il est propulsé par une turbine à gaz LM 2500 ; son armement principal est constitué de missiles mer-mer,

- le JET FOIL, construit également par Boeing, est un hydroptère commercial de 115 tonnes, de vitesse 48 nœuds qui peut transporter 200 passagers environ.

En France, une petite plate-forme expérimentale de 4,5 tonnes - 50 nœuds, le $\mathrm{H} 890$, a été réalisée par l'Aérospatiale (photo 3 ).

Les développements futurs sont possibles dans deux domaines, ceux de la taille et de la vitesse.

L'extrapolation en taille des hydroptères (photo 4) pose surtout des problèmes de développements technologiques, en particulier pour les servocommandes de pilotage, la propulsion et l'usinage des ensembles porteurs. Un point critique est celui des transmissions de puissance dans le cas d'une propulsion par hélice. Pour un hydroptère de 1000 tonnes - 55 nœuds, propulsé par deux hélices, la puissance à transmettre est de $16 \mathrm{MW}$ par ligne d'arbres avec un rapport de vitesse de rotation 4/1. De tels développements ont été entrepris aux USA par la Société Curtiss-Wright et ont conclu à la faisabilité de telles transmissions pour des puissances allant jusqu'à 40000 chevaux : des prototypes ont tourné sur banc.

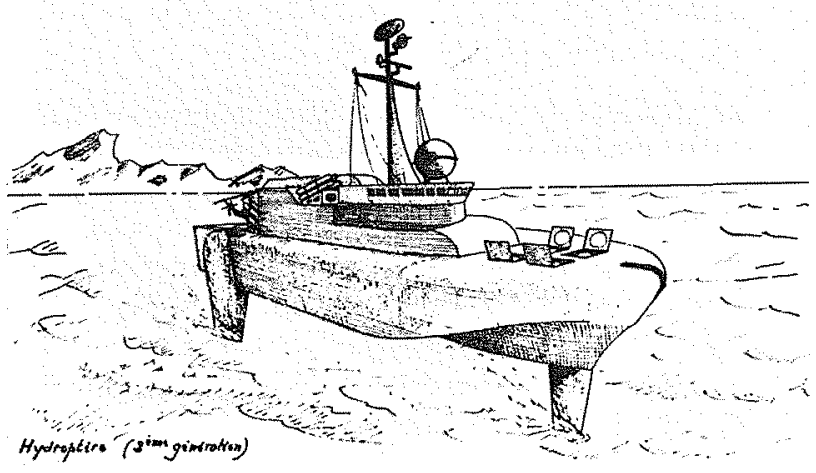

Photo 4 - Hydroptère lourd, 2000 tonnes. Vue d'artiste. 
Un élément favorable pour l'extrapolation en taille est une augmentation de la finesse avec le tonnage; pour un gros hydroptère, vue l'immersion en valeur absolue des ailes, les contraintes en cavitation sont moins sévères et la trainée relative de la part immergée de la jambe est plus faible. Une finesse globale de 10 peut être retenue pour un hydroptère de 200 tonnes - 50 nœuds ; une finesse globale de 14 à 15 est accessible pour un hydrop. tère de 1500-2000 tonnes de même vitesse maximale.

La Société Boeing a étudié en 1974 un avant-projet d'hydroptère lourd de 1600 tonnes, le DEH, de vitesse maximale 50 nœuds et à capacité océanique : cette étude a conclu à la faisabilité d'un tel hydroptère sans révolution technologique.

Le deuxième progrès possible est la vitesse. Les profils subcavitants ne permettent pas de dépasser 55 ncuds. Les profils ventilés, qui en sont encore au stade de la recherche, permettraient d'atteindre une vitesse de 70 à 75 nœuds : le niveau de connaissance, surtout pour les configurations tridimensionnelles, n'a pas encore atteint celui des précédents. Les profils supercavitants et superventilés donnent l'accès aux vitesses supérieures. Ce gain en vitesse ne va pas sans une dégradation de la finesse globale des ensembles porteurs : on peut espérer seulement 8 à 11 pour les ailes ventilées et encore moins pour les ailes superventilées. Ces hydroptères très rapides auront donc une autonomie moindre à grande vitesse et leur extrapolation en taille est moins évidente.

\section{Les navires à effet de surface}

La troisième famille de navires non conventionnels est celle des engins dont la sustention est aérostatique ou aérodynamique. Ils sont regroupés sous le nom de navires à effet de surface (NES).

La substentation aérostatique regroupe l'ensemble des véhicules à coussin d'air. Parmi ceux-ci, deux types sont à distinguer (fig. 4) :

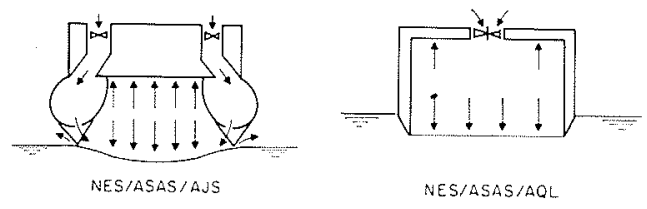

Figure 4

- les aéroglisseurs amphibies à jupes souples ou "hovercrafts" (AJS) : ces véhicules sont peu denses (pression de coussin de l'ordre de $0,25 \mathrm{~T} / \mathrm{m}^{2}$ ) et capables de très grandes vitesses par mer calme ( 70 à 80 nœuds). Ils sont développés dans la gamme de tonnages jusqu'à 200-250 tonnes, tels les SRN 4 britanniques et le N 500 Français ;

-- le deuxième type est celui des aéroglisseurs à quilles laterales (AQL) : l'ambition de cette formule est identique en vitesse, mais les tonnages visés sont d'un ordre de grandeurs plus élevé (plus de 2000 tonnes, voire 10000 tonnes et plus dans le futur).
Le NES/AQL se distingue de l'aéroglisseur amphibie par les caractéristiques suivantes :

- deux quilles latérales confinent le coussin sur les côtés ; seuls l'avant et l'arrière sont justiciables de procédés comparables à ceux des hovercrafts, tels les jupes souples, pour fermer le coussin d'air.

- les pressions de coussin d'air sont beaucoup plus élevées (1 à $1,5 \mathrm{~T} / \mathrm{m}^{2}$ ), ce véhicule est donc beaucoup plus dense : un AQL de 2000 tonnes mesurerait environ $70 \mathrm{~m}$ de long et $30 \mathrm{~m}$ de large ; à titre de comparaison, le $\mathrm{N} 500$ mesure $50 \mathrm{~m}$ sur $23 \mathrm{~m}$ pour une masse totale de 250 tonnes.

La trainée en fonction de la vitesse, pour un tonnage donné, dépend fondamentalement de deux paramètres, le rapport pression de coussin/longueur et l'élancement longueur/largeur.

Deux cas extrêmes de véhicules peuvent être envisagés.

Un NES/AQL, de faible élancement $(2$ à 2,5$)$ et de forte pression coussin est le plus avantageux pour les grandes vitesses ( 80 nœuds), mais présente une bosse de trainée très marquée à faible vitesse (40 nœuds pour un 4000 tonnes), pénalisante pour le système propulsif, qui correspond au déjaugeage : tout le domaine des vitesses immédiatement supérieures à la vitesse de déjaugeage correspond à une situation instable pour le véhicule et il n'est pas possible d'y rester de façon durable.

Un NES/AQL de grand élancement (5 et davantage) n'a pas de bosse de trainée équivalente, a un domaine continu de vitesse, mais sa vitesse maximale est plus faible, sa finesse étant moins bonne à grande vitesse.

Le choix entre ces deux sous-filières dépend essentiellement du profil de la mission du véhicule.

Deux prototypes de 100 tonnes, construits par Bell (photo 5) et Aérojet Général ont été essayés au cours des trois dernières années aux USA. Ils ont atteint des vitesses supérieures à 80 nœuds. Les études d'un NES/AQL de 3000 tonnes ont été commencées en 1977.

Les problèmes majeurs posés par le NES/AQL sont : -- le devis de poids : la puissance installée est très

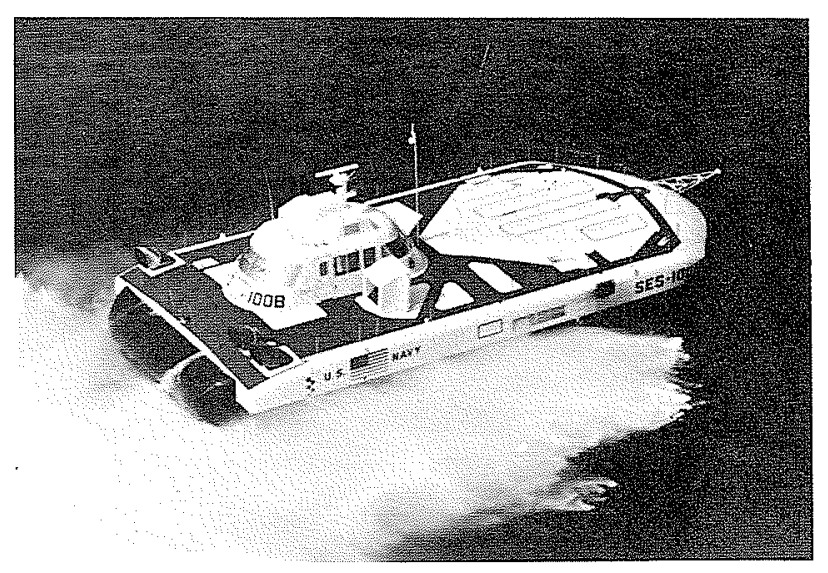

Photo 5 - NES. Bell Aerospace 100B (photo US Navy). 
élevée. Un NES/AQL de 4000 tonnes et de vitesse supérieure à 60 nœuds doit avoir une puissance totale, pour la propulsion et la sustentation, de l'ordre de $200000 \mathrm{ch}$. La consommation horaire, à la vitesse maximale, est de 40 tonnes. Pour obtenir 50 heures d'autonomie, le poids de carburant à embarquer est égal à la moitié du déplacement en charge. Compte tenu du poids de l'appareil propulsif et des autres équipements, le poids de la structure doit être inférieur à $28 \%$ du déplacement. Il y a donc un problème de réalisation d'une grande structure en alliage léger ;

- la tenue à la mer : surtout pour les NES/AQL de faible élancement, les performances, vitesse ou tranquillité de plate-forme, se dégradent très vite avec l'état de la mer. La houle agit comme un piston sur le coussin d'air et provoque des accélérations verticales excessives. $\mathrm{Ce}$ point est critique non seulement pour les performances intrinsèques de l'appareil, mais également pour l'endurance de l'équipage. L'influence de la pente de la caractéristique débitpression des ventilateurs de sustentation est déterminante pour les accélérations : l'idéal serait d'aboutir à une caractéristique aussi plate que possible, un réglage actif du débit de sustentation par des aubages orientales ou le réglage de la géométrie des stators ne sont pas exclus. Un contrôle des surpressions du coussin par des évents est peu envisageable pour des grands véhicules, vu les pertes qu'il entraîne;

- les fermetures avant et arrière : par rapport aux aéroglisseurs amphibies, les difficultés nouvelles sont liées aux pressions de coussin plus élevées et aux dimensions.

L'évolution ultime du navire non conventionnel est le véhicule à sustentation aérodynamique. On peut le considérer comme résultant d'une évolution à partir des NES/AQL (fig. 5).

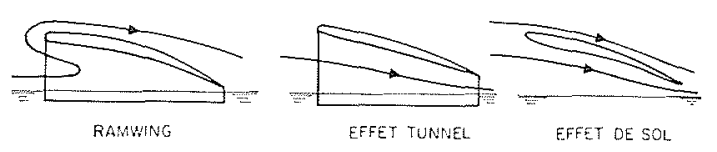

Figure 5

Si on supprime sur un NES/AQL la fermeture avant, on obtient à vitesse élevée un véhicule baptisé Ramwing aux USA. Les quilles latérales peuvent être totalement émergées.

Si on supprime en outre la fermeture arrière, la portance est assurée par effet tunnel. Si enfin on supprime les quilles latérales, on obtient un véritable avion volant en effet de sol.

Il n'y a pratiquement pas de réalisations dans le monde occidental, les recherches du Docteur Lippish mises à part. L'URSS a consacré l'effort le plus important à cette filière. Un véhicule, appelé Ekranoplane, aurait volé sur la mer Caspienne : cet engin de 500 tonnes et de 38 mètres d'envergure, serait capable de voler à plus de 200 nceuds à des altitudes comprises entre 12 et 23 mètres.

Les inconnues principales sur ce concept sont la tenue sur houle et la manœuvrabilité ; quels sont en particulier les ordres de grandeurs des efforts induits par les vagues, et un pilotage est-il possible?

L'intérêt essentiel de la formule est sa finesse (des valeurs de 30 sont annoncées) et la charge utile très élevée.

Le déplacement sur l'eau à vitesse réduite, moins de 100 nœuds, et les phases transitoires sont difficiles à imaginer autrement que par des concepts hybrides.

Les formules hybrides de navires non conventionnels n'ont fait jusqu'ici l'objet que d'études prospectives.

\section{La propulsion des navires non conventionnels}

L'exposé qui suit est limité aux propulsions marine ou mixte. La propulsion aérienne est utilisée avec succès sur les aéroglisseurs amphibies, dont la faible compacite s'accommode bien d'hélices de grand diamètre, faiblement chargés et de haut rendement. Ce n'est pas le cas des autres formules, y compris les NES/AQL où l'implantation de plusieurs hélices de 10 mètres de diamètre et plus pose des difficultés insurmontables : dans ce cas particulier, une propulsion par turbosoufflantes à très grand taux de dilution, jusqu'à 30, mérite d'être étudiée. Il faut noter qu'une propulsion aérienne n'est pas envisageable sur le NES/AQL si celui-ci doit mettre en cuvre des aéronefs.

Seul le BSCI ne pose pas de problème au plan du propulseur : les vitesses visées et l'immersion autorisent l'emploi d'hélices marines classiques de grand diamètre à haut rendement.

Pour tous les autres types, il a fallu développer des propulseurs spécifiques. Le premier arrivé à maturité sur le plan technologique est l'hydrojet. Ce propulseur est celui qui équipe les hydroptères Boeing et celui qu'on retrouve le plus souvent dans les projets de NES/AQL. Des études sont en cours aux USA pour développer des hydrojets de puissance unitaire de 50000 chevaux. Il est constitué schématiquement d'une prise d'eau, d'un circuit d'aspiration à l'intérieur du navire, d'une pompe et d'une buse de rejet.

Les deux organes les plus critiques sont la pompe et la prise d'eau. Les pompes ont atteint un niveau élevé de développement (avec un rendement supérieur à 90\%) ; les prises d'eau sont délicates à concevoir lorsque le véhicule présente une bosse de déjaugeage. Le débit dans l'hydrojet doit alors être sensiblement le même à vitesse de déjaugeage et à vitesse maximale, ce qui impose le recours à une géométrie variable pour la prise d'eau.

Globalement, l'hydrojet se présente comme un propulseur bien adapté aux navires non conventionnels, mais d'un rendement moyen (50 à $55 \%$ ), peu susceptible de grosses améliorations dans l'avenir et d'un poids au cheval élevé $(1,5 \mathrm{~kg} / \mathrm{ch})$ compte tenu de la quantité d'eau embarquée à bord.

Il permet d'avoir une transmission en ligne, avec un rapport de réduction faible compte tenu de la vitesse de rotation plus élevée des pompes par comparaison à celles des hélices. 
Des concurrentes de l'hydrojet, au moins pour les hydroptères, sont les nouveaux types d'hélices développées parallèlement aux nouveaux profils d'ailes d'hydroptères :

- les hélices ventilées entre 40 et 60-65 nœuds

- les hélices superventilées et supercavitantes.

Leur mérite essentiel est leur plus haut rendement. Un rendement propulsif maximum de 60-62 \% peut être atteint avec une hélice supercavitante. De telles hélices équipent un des hydroptères expérimentaux américains, le Plainview de 320 tonnes. Pour des hydroptères de gros tonnage, l'hélice présente sur l'hydrojet l'avantage déterminant de leur donner une autonomie en rapport avec leur taille.

Cette évolution est moins évidente pour les NES/ AQL : des hélices semi-immergées superventilées (dont l'arbre et le moyeu sont dénoyés) sont étudiées aux USA, un des prototypes de 100 tonnes en ayant été équipé. Elles ne semblent pas toutefois avoir atteint un niveau de développement comparable à celui de l'hydrojet.

Une autre propulsion possible est une propulsion mixte air-eau, la propulsion biphasique, mettant à profit le déplacement du navire non conventionnel entre les deux fluides. De très nombreuses études ont été consacrées à ce mode de propulsion depuis les trois dernières décennies et on peut se demander pourquoi elles n'ont pas encore abouti. Deux classes sont à distinguer dans la propulsion biphasique :

- la propulsion par brouillard où le débit d'eau est pulvérisé dans le flux froid d'une turbosoufflante, le rapport entre les débits masse eau/gaz est alors sensiblement de l'ordre de 10 ;

- la propulsion par émulsions, où au contraire le débit de gaz est injecté dans le débit d'eau, le rapport entre les débits masse eau/gaz étant alors plus voisin de 1000 .

Les études consacrées aux USA à la propulsion par brouillard, "le mist-jet", n'ont pas encore abouti à des réalisations opérationnelles. Plusieurs causes à cet état de fait sont à relever :

-- ces écoulements mixtes sont difficiles à modèliser et les premières évaluations d'application aux navires non conventionnels ont largement sous-estimé les pertes diverses,

- ces évaluations ont été faites pour des véhicules très rapides présentant une bosse de déjaugeage ; il existe un problème réel d'adaptabilité de la propulsion par brouillard aux faibles vitesses, le débit d'eau devant être augmenté dans un rapport 5 à 10 par rapport à la croisière, et aboutissant à un surdimensionnement des circuits,

-- pour diminuer les pertes, l'eau doit être pulvérisée très finement : le problème posé est de pulvériser des débits d'eau de quelques tonnes/seconde en goutelettes de moins de $1 / 10$ de $\mathrm{mm}$ de diamètre.

De toute façon, les poussées spécifiques à attendre de la propulsion par brouillards sont faibles $(1$ à 2 tonnes $/ \mathrm{m}^{2}$ ) et son avenir doit être recherché dans une intégration aussi poussée que possible au véhicule : un exemple de recherche dans ce sens est le système
WAVE "Water Augmented Vehicle", étudié aux USA, où le brouillard créé par les embruns au bas des jupes d'un aéroglisseur est récupéré dans des conduits intégrés au coussin d'air.

Des problemes équivalents ont surgi dans les études de propulseurs à émulsions. Compte tenu des rapports de masse de deux phases, des poussées spécifiques plus fortes $\left(5\right.$ à $\left.10 \mathrm{~T} / \mathrm{m}^{2}\right)$ peuvent être espérées. Les problèmes de poussée à basse vitesse et d'élimination des pertes par intégration au véhicule de modélisation et de transposition d'essais à petite échelle subsistent.

En dépit des difficultés rencontrées, la poursuite d'études sur la propulsion biphasique est justifiée : sur un NES/AQL, elle permettrait de supprimer les transmissions et le propulseur, d'où un avantage manifeste pour le poids, voire la fiabilité et la maintenance. Un rendement acceptable reste impératif : par exemple, pour un NES/AQL de 4000 tonnes, si la propulsion biphasique permettait, à titre de simple hypothèse, de diviser par deux le poids de l'appareil moteur par rap. port à la solution hydrojet, il faudrait que la propulsion biphasique ait un rendement global de l'ordre de $45 \%$ pour conserver la même distance franchissable sans pénaliser les autres éléments du devis de poids.

Un dernier aspect est celui de la source de puissance : pour les navires non conventionnels de faible tonnage, une analyse était à faire entre le moteur Diesel et la turbine à gaz : en effet, le Diesel bien que plus lourd, avait l'avantage de consommations spécifiques moins élevées. La turbine à gaz l'emporte toutefois dans les réalisations les plus récentes, vus les progrès accomplis dans le domaine de la consommation. Pour des navires non conventionnels de fort tonnage, il n'y a pas d'autre choix que les turbines à gaz de forte puissance unitaire (20 à 40000 chevaux) et dont les consommations spécifiques sont de l'ordre de 180 à $200 \mathrm{~g}$ cheval-heure.

La propulsion nucléaire serait bien évidemment un avantage déterminant pour des NES/AQL de très gros tonnage (plus de 4000 tonnes) : les réacteurs à eau pressurisé développés jusqu'ici pour les navires classiques et les sous-marins, ne sont pas transposables; pour un NES/AQL de 4000 tonnes, le poids de la chaufferie nucléaire seule serait pratiquement égal au déplacement total en charge. La propulsion nucléaire sera envisageable que dans des techniques où le poids au cheval sera diminué d'un ordre de grandeur : ceci est envisageable avec des réacteurs légers à haute température par exemple, mais il est difficile de prédire leur horizon d'aboutissement pour des applications marines.

Ce bref tour d'horizon sur les navires non conventionnels montre qu'ils sont arrivés à maturité dans la gamme des déplacements modestes, moins de 300 tonnes, et pour des vitesses de l'ordre de 50 à 70 ncuds. Une extrapolation en taille, vers les milliers de tonnes, et en vitesse pour approcher les 100 nœuds, nécessite des études nouvelles et surtout des développements technologiques qui jusqu'ici n'ont été véritablement entrepris qu'aux Etats-Unis. L'état de l'art en France est plus modeste, la capacité de réaliser la première génération de ces véhicules dans les tonnages faibles étant toutefois atteinte. 


\section{Discussion}

Président: M. A. CASTERA

M. le Président remercie M. LE TALLEC pour son exposé qui montre clairement qu'en matière de véhicules à grande et à très grande vitesse on le trouve encore en pleine gestation et il poursuit :

Quelles sont les perspectives d'avenir des différents types aujourd'hui en présence?

Pour les batiments de surface à coque immergée (B.S.C.I.), il semble qu'il y ait un créneau intéressant pour des tonnages compris entre 4000 et 10000 tonnes et des vitesses allant jusqu'à une quarantaine de nœuds. Encore faudrait-il que les cas d'utilisations pratiques apparaissent plus clairement qu'à l'helure actuelle.

J'exclus le cas des navires à sustentation aérodynamique qui relèvent du domaine de l'aéronautique et celui des hydroptères de premiere génération à ailes fixes qui ne sont guère commercialement utilisables que dans des zones très abritées. Il y a d'ailleurs pour ce type d'hydroptère une expérience de près d'une trentaine d'années puisque les premiers sont apparus, en Italie, aux environs de 1950.

Demeurent donc en compétition les hydroptères de deuxième génération - à l'heure actuelle, on s'accorde à leur assigner, au moins temporairement, une limite de tonnage de l'ordre de 2000 tonnes - et les N.E.S.A.Q.L. (navires à effet de surface a quilles latérales). En haute mer, on reproche, en effet, aux navires à coussin d'air des formes peu aérodynamiques et aux modèles amphibies une certaine instabilité latérale. Je dois dire, que le terme "coussin d'air" est assez mal choisi car dès que la mer force un peu, il s'agit d'un coussin fort mal rembourré.

De caractère plus militaire, les N.E.S.A.Q.L. (à quilles latérales) paraissent offir des perspectives intéressantes, à conditions d'atteindre des tonnages notables.

M. LE TALLEC a souligné que la construction des navires non conventionnels fait appel à des technologies qui relevent davantage de la construction aéronautique que de la construction navale classique : il en résulte des prix de revient élevés.

A noter, qu'on a assisté à un curieux chassé-croisé dans le développement des navires non conventionnels; à une certaine époque, le secteur militaire semblait attendre le développement d'un Marché civil pour se lancer dans l'aventure ; aujourd'hui, à l'inverse, c'est plutôt le secteur civil qui espère des développe- ments militaires qui permettraient à moindre frais la mise au point de versions civiles.

Je voudrais attirer, ici, votre attention sur ce que je considère comme une assez remarquable réussite, à la suite des efforts qui ont été faits, en France, pour le développement des ébauches de navires non conventionnels : il s'agit de l'hydroptère "plateforme d'essai" dont une diapositive vous a été projetée tout à l'heure. Dans le domaine des hydroptères de deuxième génération a laquelle appartient cet engin - aux ailes complètement pilotées - la petite taille conduit en ce qui concerne les problemes de fustentation hydrodynamique et les problèmes de pilotage automatique aux conditions les plus critiques. M. LE TALLEC a indiqué, par exemple, que, pour un engin de cette sorte, essayé sur l'Etang de Berre, avec des houles courtes présentant des creux de 80 centimètres au maximum, la fréquence de rencontre de l'engin avec les crêtes de vague, peut être de l'ordre de 5 par seconde; autrement dit, le pilotage automatique pour le braquage des ailes doit intervenir 5 fois par seconde pour obtenir une trajectoire horizontale.

Technologiquement, ceci est assez sévère, pour les organes de commandes des ailes, même à cette échelle réduite ; les charges sur les ailes de cet engin sont, en effet, de l'ordre de 5 tonnes par $\mathrm{m}^{2}$, c'està-dire, 5 fois celles admises pour des ailes d'avion.

Certains d'entre vous ont peut-être vu, le H-890, à l'exposition navale du Bourget et constaté que la surface totale des ailes n'était que de l'ordre du mètre carré. En raison de sa haute résistance mécanique, il avait été envisagé d'utiliser le titane pour réaliser les ailes du H-890. Mais, la "fréquence de rencontre" de l'ordre de 5 hertz, aurait alors été très voisine de la fréquence propre de l'ajle, et on a dû revenir à un acier inox qui a au moins l'avantage d'avoir un module d'élasticité beaucoup plus élevé que celui du titane.

Je remercie encore $M$. LE TALLEC de nous avoir initié à un domaine trés nouveau et passionnant et je souhaite, qu'à l'échelle française, les perspectives de développement des navjres non conventionnels se révèlent favorables, sinon dans l'immédiat, au moins à moyen terme.

En l'absence d'autre intervention, M. le Président donne la parole à $M$. le Professeur DODU pour l'exposé de la Communication qu'il a établie en collaboration avec MM. MICHEL et ROWE de l'Institut de Mécanique de Grenoble. 